\title{
Current Diffusion and Normal Zone Propagation Inside the Aluminum Stabilized Superconductor of ATLAS Model Coil
}

\author{
E. W. Boxman, M. Pellegatta, A. V. Dudarev, and H. H. J. ten Kate
}

\begin{abstract}
The normal zone propagation inside the $B_{0}$ model coil of ATLAS Toroidal magnet has been measured over a large range of applied currents. Typical values for the longitudinal propagation vary from 0.3 to $15 \mathrm{~m} / \mathrm{s}$ at 8 and $24 \mathrm{kA}$, respectively. A new analytical expression for the longitudinal quench propagation inside superconducting cables is presented. It describes the propagation inside superconducting wires as well as the propagation inside large stabilized superconductors. It is found that in the limit case of high currents, the stabilizer functions only as a heat-sink. The model is compared to the experimental data and a good correlation is found.
\end{abstract}

Index Terms-Current distribution, stability, superconductors.

\section{INTRODUCTION}

C URRENTLY, the largest superconducting magnet system of the world is under construction. It is the magnet system for the ATLAS [1] detector that will be used in the new proton-proton collider LHC at CERN. Since the magnets and the stored energy are large, also the superconductors are scaled up to a very large size, typically with a cross-section of a few hundreds square millimeters. When a disturbance initiates a transition to the normal state in such conductor (quench), the conductor should be able to carry the current for a relatively long time period. This is because of the time to detect a quench and the time for ramping down the current is long (minutes) in such magnet systems. For this reason, a relatively large cladding of highly pure Aluminum is placed around the NbTi superconductor in order to protect the conductor from overheating. In this way the current can redistribute into the normal conducting Aluminum stabilizer, dissipating less energy than without the cladding. This scaling causes particular problems in modeling of the superconductors with respect to the quench behavior and safety characteristics. During the current re-distribution, the current density is not uniform in the cross-section of the conductor which makes the calculations of the growth of the resistive volume complex. Another aspect is the temperature and the magnetic field dependences of the material properties. Together, for calculating the normal zone

Manuscript received August 6, 2002. This work was supported in part by the Netherlands Technical Foundation (STW).

E. W. Boxman is with CERN, Geneva, Switzerland and also with the Faculty of Applied Physics, University of Twente, 7500 AE Enschede, The Netherlands (e-mail: E.W.Boxman@tn.utwente.nl).

A. V. Dudarev and H. H. J. ten Kate are with CERN, Geneva, Switzerland. Digital Object Identifier 10.1109/TASC.2003.812866 propagation, a set of nonlinear partial differential equations are to be solved [2]-[4]. Here, an analytical expression for the normal zone propagation is given considering also the current diffusion into the Aluminum. This could make modeling of the superconductors with respect to the quench behavior and safety characteristics, more practical.

\section{NORMAL ZONE PROPAGATION}

\section{A. At Low and High Currents, the Two Limit Cases}

The standard calculations for the normal zone propagation in a thin wire is depending on the current density $J\left[\mathrm{~A} / \mathrm{m}^{2}\right]$, the specific heat of the composite $C_{m}(B, T)[\mathrm{J} / \mathrm{kgK}]$, the density of the material $\gamma\left[\mathrm{kg} / \mathrm{m}^{3}\right]$, the longitudinal heat conduction coefficient $\kappa(B, T)[\mathrm{W} / \mathrm{mK}]$, the temperature difference between the current sharing temperature $T_{\mathrm{cs}}(B, J)$ and the bath temperature $T_{0}[\mathrm{~K}]$ and the electrical resistivity of the matrix material $\rho(B, T)[\Omega \mathrm{m}][5]$. In the Atlas type superconducting cable, the current density is not trivial due to the diffusion of the current into the stabilizer. However at low currents, where the velocity is expected to be small, the current is distributed approximately uniformly over the whole cross-section of the stabilizer. On the other hand, due to the high purity of the Aluminum the heat conductivity of this cladding is much higher compared to the heat conductivity of the normally used copper matrix of the superconductor. Therefore, the transfer of heat to the front of the normal zone is expected to be transported by the cladding. In this case, the characteristic velocity in the adiabatic approximation at low currents becomes

$$
v_{\mathrm{low}} \approx \frac{I}{\gamma C_{m} A_{\mathrm{Al}}}\left(\frac{\rho_{\mathrm{Al}} \cdot \kappa_{\mathrm{Al}}}{T_{\mathrm{cs}}-T_{0}}\right)^{1 / 2}[\mathrm{~m} / \mathrm{s}] .
$$

At high currents, the heat dissipation that contributes to the normal zone front is mainly formed inside the superconductor cable and not in the cladding resulting in an increase of the propagation. When the area of stabilizer $\left(A_{\mathrm{Al}}\right)$ is large compared to the area of the superconductor $\left(A_{\mathrm{NbTi}}\right)$, the normal zone propagation at high currents can be described by

$$
v_{\mathrm{high}} \approx \frac{I}{\gamma C_{m} \sqrt{A_{\mathrm{NbTi}} A_{\mathrm{Al}}}}\left(\frac{\rho_{\mathrm{Al}} \cdot \kappa_{\mathrm{Al}}}{T_{\mathrm{cs}}-T_{0}}\right)^{1 / 2}[\mathrm{~m} / \mathrm{s}] .
$$

In this case, the Aluminum cladding is only functioning as a heat-sink. When the current is between these two extremes, the current diffusion has to be considered. 


\section{B. Current Diffusion}

In Fig. 1, an Aluminum stabilized superconductor is schematically drawn. On the right side the cable is still superconducting, on the left side the cable is in the normal state. When a disturbance occurs and the temperature rises above the current sharing temperature $T_{\mathrm{cs}}$, the current starts to diffuse into the cladding as shown in the figure.

If the current diffusion starts at $t=0$, then after a certain time the ohmic heat that is generated inside the cladding can not contribute to the normal zone propagation $(v)$ simply as the extra heat takes to much time to propagate to the front. This implies that there exists a boundary where only at one side the generated heat can contribute to the propagation. We define a normal zone length $\left(L_{\mathrm{nzf}}\right)$, which mainly contribute to the normal zone propagation. Hence:

$$
L_{\mathrm{nzf}}=v \cdot \tau_{H}[\mathrm{~m}]
$$

where $\tau_{H}$ is a characteristic time of the thermal diffusion over the length $L_{\mathrm{nzf}}$, which is given by

$$
\tau_{H}=\frac{L_{\mathrm{nzf}}^{2}}{D_{H}}=\frac{L_{\mathrm{nzf}}^{2} \gamma C_{m}}{\kappa_{\mathrm{Al}}}[\mathrm{s}]
$$

where in the case of the Atlas type stabilized superconductors, $D_{H}$ is the thermal diffusivity of the Al. $\left(\mathrm{m}^{2} \mathrm{~s}^{-1}\right)$. By using (3) and (4), $\tau_{H}$ can be written as

$$
\tau_{H}=\frac{\kappa_{\mathrm{Al}}}{\gamma C_{m} v^{2}}[\mathrm{~s}] .
$$

In this kind of rectangular conductors as depicted in Fig. 2, the current diffusion takes place in two directions with different diffusion lengths. This implies that there are two different characteristic diffusion time constants. The characteristic time for the current diffusion in the $x$ direction is:

$$
\tau_{d x}=\frac{L_{d x}^{2} \mu_{0}}{\pi^{2} \rho_{\mathrm{Al}}}=\frac{(a-c)^{2} \mu_{0}}{4 \pi^{2} \rho_{\mathrm{Al}}}[\mathrm{s}]
$$

and for the $y$ direction:

$$
\tau_{d y}=\frac{L_{d y}^{2} \mu_{0}}{\pi^{2} \rho_{\mathrm{Al}}}=\frac{(b-d)^{2} \mu_{0}}{4 \pi^{2} \rho_{\mathrm{Al}}}[\mathrm{s}] .
$$

In the case of the Atlas Barrel Toroid superconductor, the typical values for this time constant in the $x$-direction lay between $0.5-1.5 \mathrm{~s}$.

By assuming that the current diffusion is according to an exponential behavior, then for the effective area occupied by the current can be written as

$$
a_{\mathrm{Al}}(t)=\begin{aligned}
& {\left[\left(a-a_{0}\right) \cdot\left(1-e^{-\frac{t}{\tau_{d x}}}\right)+c \sqrt{\alpha}\right] \cdot\left[\mathrm{m}^{2}\right],} \\
& {\left[\left(b-b_{0}\right) \cdot\left(1-e^{-\frac{t}{\tau} d y}\right)+d \sqrt{\alpha}\right]}
\end{aligned}
$$

with $\alpha$ the Superconductor/Normal conductor ratio. By using the boundary conditions

$$
v \rightarrow 0 \Rightarrow a_{\mathrm{Al}}(\infty)=A_{\mathrm{NbTi}}=\alpha \cdot c d,
$$

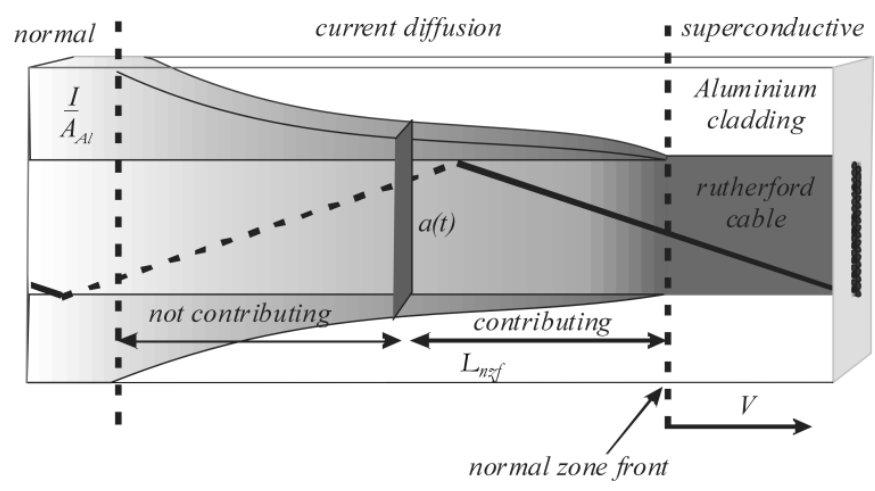

Fig. 1. Schematic presentation of the current diffusion in stabilized superconductors.

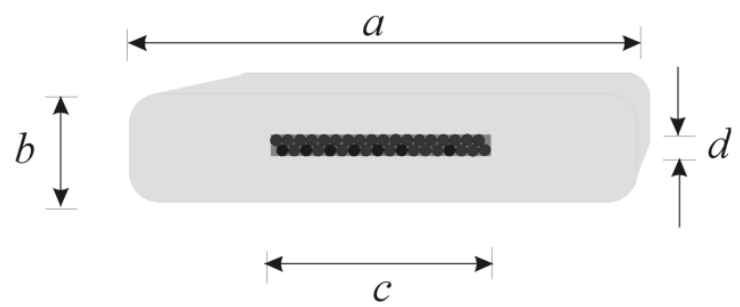

Fig. 2. The layout of the conductors used in the Atlas superconducting magnet system. In the middle the Rutherford shaped cable is shown.

$v \rightarrow \infty \Rightarrow a_{\mathrm{Al}}(0)=A-A_{\mathrm{Cu}}=A-(1-\alpha) c d$, which give the constants

$$
\begin{aligned}
a_{0}= & \frac{(a d+c b)}{2 d} \\
& -\frac{\sqrt{(a d+c b)^{2}-4 c^{2} d^{2}(1-\alpha)}-2 c d \sqrt{\alpha}}{2 d}[\mathrm{~m}]
\end{aligned}
$$

and

$$
b_{0}=\frac{d}{c} a_{0}[\mathrm{~m}] .
$$

\section{Heat Balance Equation}

Starting form the heat balance equation and assuming that the heat conductivity is mainly due to the relatively high heat conductivity of the pure Aluminum, the longitudinal propagation velocity can be extracted:

$$
\begin{aligned}
\frac{\partial}{\partial z}\left(k A_{\mathrm{Al}} \frac{\partial T}{\partial z}\right) & -\gamma C_{m} A \frac{\partial T}{\partial t} \\
& -h P\left(T-T_{0}\right)+G \cdot a(t)=0[\mathrm{~W} / \mathrm{m}],
\end{aligned}
$$

with the ohmic heat generation

$$
G=\frac{I^{2} \rho_{\mathrm{eff}}}{a(t)^{2}}\left[\mathrm{~W} / \mathrm{m}^{3}\right] .
$$

Here, the $\rho_{\text {eff }}$ is the effective electrical resistivity. For simplicity reasons, the specific heat of the Rutherford cable is taken equal to the specific heat of the Aluminum. By using a network model of two parallel resistances, the effective resistance of the conductor becomes

$$
\rho_{\mathrm{eff}}=\frac{a(t) \rho_{\mathrm{Cu}} \cdot \rho_{\mathrm{Al}}}{A_{\mathrm{Cu}} \rho_{\mathrm{Al}}+a_{\mathrm{Al}}(t) \rho_{\mathrm{Cu}}}[\Omega \mathrm{m}] .
$$


In this equation, $a_{\mathrm{Al}}(t)$ is the area of the Aluminum including the NbTi. Solving the differential equation following (2) then, in the adiabatic case the longitudinal propagation velocity becomes

$$
\begin{aligned}
& v=\frac{I}{\gamma C_{m} f(t)} \cdot\left(\frac{\rho_{\mathrm{Cu}} \cdot \rho_{\mathrm{Al}} \cdot \kappa_{\mathrm{Al}}}{\left(T_{\mathrm{cs}}-T_{0}\right)}\right)^{1 / 2}[\mathrm{~m} / \mathrm{s}], \\
& f(t)=\sqrt{\frac{\left(A^{2}\right)\left(A_{\mathrm{Cu}} \rho_{\mathrm{Al}}+a_{\mathrm{Al}}(t) \rho_{\mathrm{Cu}}\right)}{A-A_{\mathrm{ruth}}}}\left[\mathrm{m}^{2}\right],
\end{aligned}
$$

where $A$ is the total cross-section of the cable, $A_{\text {ruth }}$ is the area of the Rutherford cable and $A_{\mathrm{Cu}}$ the copper area of the Rutherford cable. By using (8) at $t=\tau_{h}$, a implicit formula is to be solved. However, an explicit solution can be made when the $\tau_{h}<\tau_{d}$. By using the Taylor expansion of the area $a_{\mathrm{Al}}(t)$ around $t=0$, i.e., when the heat diffusion is much faster than the electromagnetic diffusion. This is when

$$
v^{2}>\frac{\kappa_{\mathrm{Al}}}{\gamma C_{m} \tau_{d}}\left[\mathrm{~m}^{2} / \mathrm{s}^{2}\right]
$$

The explicit expression for the normal zone velocity can be written as

$$
v=\left[R_{I v}\left(\left(v_{0}-v_{d}\right)\right)\right]^{1 / 2}[\mathrm{~m} / \mathrm{s}]
$$

with

$$
\begin{aligned}
& v_{0}=\frac{I^{2}\left(A-A_{\mathrm{ruth}}\right)}{\gamma C_{m} A^{2}\left(T_{\mathrm{cs}}-T_{0}\right)}[\mathrm{m} / \mathrm{s} \Omega], \\
& v_{d}=\frac{4 \pi^{2}}{\mu_{0}}\left(\frac{\left(b-b_{0}\right) c \sqrt{\alpha}}{(b-d)^{2}}+\frac{\left(a-a_{0}\right) d \sqrt{\alpha}}{(a-c)^{2}}\right)[\mathrm{m} / \mathrm{s} \Omega],
\end{aligned}
$$

and

$$
R_{I v}=\left(\frac{\rho_{\mathrm{Cu}} \rho_{\mathrm{Al}} \kappa_{\mathrm{Al}}}{\gamma C_{m}\left(A_{\mathrm{Cu}} \rho_{\mathrm{Al}}+\left(A_{\mathrm{NbTi}}\right) \rho_{\mathrm{Cu}}\right)}\right)[\Omega \mathrm{m} / \mathrm{s}] .
$$

In this expression only the sizes of the conductor and the material properties are included. Finally, as the electrical resistivity of the copper matrix is much larger than the electrical resistivity of the Aluminum, then the two limit cases of (14) at low currents and high currents become equal to (1) and (2), respectively.

\section{MEASUREMENT Results}

During the tests of the ATLAS $B_{0}$ toroid model coil, the longitudinal propagation was measured for a wide range of currents. In Fig. 3, the position of the pickup coils and voltage taps are presented schematically [10]. The normal zone was initialized by discharging a capacitor into a $100 \times 50 \mathrm{~mm}^{2}$ heater situated on the most inner turn of the magnet. The time constant of the heat pulse was $7 \mathrm{~ms}$. The pickup coils chosen in the measurement are located as far as possible from the heater at a distance of $2.76 \mathrm{~m}$ and $5.85 \mathrm{~m}$. Measurements close to the heater would lead to errors in the measurements. The longitudinal propagation is calculated using the time information between two pickup coil signals. The coil is indirectly cooled by liquid helium, which justifies the adiabatic assumption.

In Fig. 4, the results for the longitudinal velocity depending on the magnet current in the $B_{0}$ model coil are depicted. The solid line is the result of the calculation of (14). The dashed line

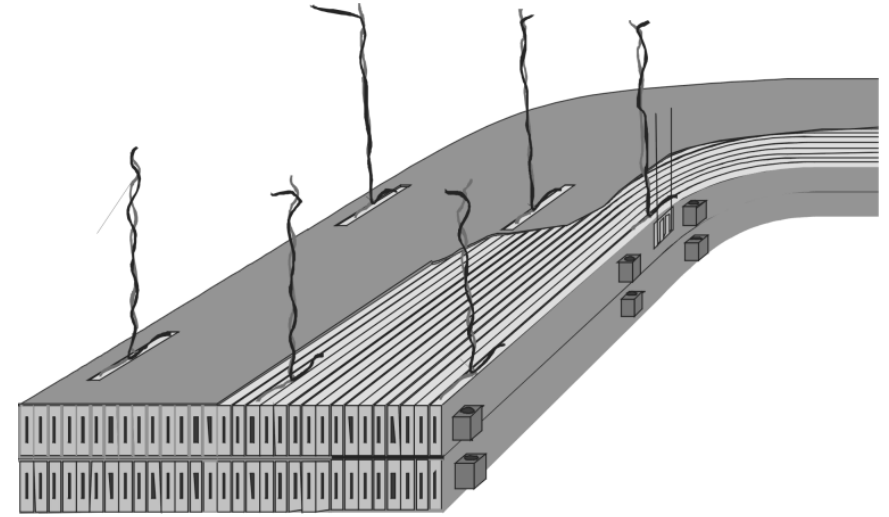

Fig. 3. Position of the voltage taps and the pickup coils in the $B_{0}$ model coil for ATLAS.

are the results of the calculation using the Taylor expansion (17), which is valid when the characteristic time of the heat diffusion is lower than the characteristic time of the electromagnetic diffusion. In addition, the two limit cases for low-and high current are presented, using (1) and (2) respectively. In all the calculations the magnetic field and temperature dependence of the material properties are included. The temperature is taken at the current sharing temperature. In Tables I and II, some important values that are used in the calculations are presented. In the case of the Aluminum cladding and the copper matrix a RRR of 1100 and 80 are taken, respectively. The calculations of (14), show a good correlation with the experimental results. However at low magnet current where the velocity is relatively slow $(<2 \mathrm{~m} / \mathrm{s})$, the experimental values are lower than the calculations. This can be understood sinc in the model the cooling is not included.

\section{DISCUSSION}

In this type of magnets, the quench propagation is nearly independent of the resistivity of the copper matrix or the Aluminum cladding. Nevertheless, the magnetic field dependence is rather strong especially in the case of the high purity Aluminum. The temperature dependences and the magnetic field dependences of the material properties have important influence in modeling the quench behavior. Good knowledge of these influences is obligatory in order to get high accurate calculations of the velocities. In our calculations, the library for the material properties of the quench propagation simulation program Q-LASA is used. In the calculations the area of the NbTi is included in the Aluminum area. This is justified as the $E J$-curve of the superconductor is not linear and therefore can adjust to the electrical resistivity of the Aluminum. This becomes important at high magnet currents.

The flight of the normal-superconducting front is also calculated from the time information of the voltage taps signals. It shows similar results compared to the pick-up coil measurements. However due to the high purity of the Aluminum the beginning of the voltage rise is difficult to determine, giving larger errors in the propagation velocity. For this reason, the sharp rise of the signal coming from the pickup coils, are better suitable for calculating the normal zone propagation.

Due to the large diffusion time constants the current at the front of the normal zone is not homogeneously distributed over 


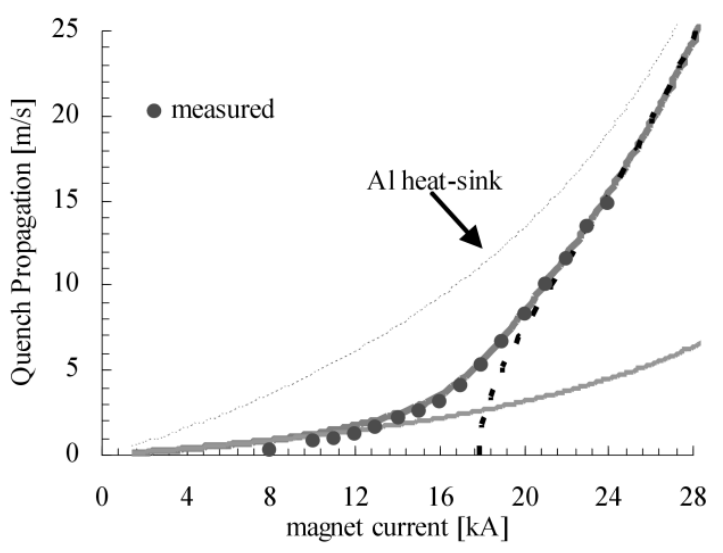

Fig. 4. The results of the measurements on $B_{0}$ model coil and the theoretical expected values using (14). The two limit cases for the low- and high current regime and the first order Taylor development (17), the dashed line, are also presented.

TABLE I

$B_{0}$ Conductor Properties USEd IN THE CALCULATiOnS

\begin{tabular}{|c|c|c|c|c|}
\hline Magnetic field & $\mathrm{T}$ & 1.0 & 2.0 & 3.0 \\
\hline Magnet Current $(I)$ & $\mathrm{kA}$ & 6.37 & 12.65 & 19.0 \\
\hline $\begin{array}{l}\text { Copper resistivity } \\
(\mathrm{Cu})(\mathrm{RRR}=80)\end{array}$ & $\underset{10^{-10}}{\mathrm{~m}}$ & 2.65 & 3.12 & 3.60 \\
\hline $\begin{array}{l}\text { Aluminium. } \\
\text { resistivity }\left({ }_{A l}\right) \\
(\mathrm{RRR}=1100)\end{array}$ & $\underset{10^{-11}}{\mathrm{~m}}$ & 5.68 & 6.03 & 6.15 \\
\hline $\begin{array}{l}\text { Aluminium heat } \\
\text { conductivity }\left({ }_{A}\right)\end{array}$ & $\begin{array}{c}\mathrm{W} / \mathrm{m} \\
\mathrm{K}\end{array}$ & 2144 & 2067 & 1991 \\
\hline Specific Heat $\left(C_{m}\right)$ & $\mathrm{J} / \mathrm{m}^{3} \mathrm{~K}$ & 2662 & 2421 & 2185 \\
\hline $\begin{array}{l}\text { Sharing temperature } \\
\left(T_{s c}\right)\end{array}$ & $\mathrm{K}^{2}$ & 8.44 & 7.9 & 7.28 \\
\hline
\end{tabular}

TABLE II

Dimensions OF THE CONDUCTOR IN $B_{0}$ MOdel COIL

\begin{tabular}{ccccccc}
\hline \hline parameter & $\mathrm{a}$ & $\mathrm{b}$ & $\mathrm{c}$ & $\mathrm{d}$ & $\mathrm{a}_{0}$ & $\mathrm{~b}_{0}$ \\
\hline$[\mathrm{mm}]$ & 57.0 & 12.0 & 27.88 & 2.30 & 2.00 & 0.165 \\
\hline \hline
\end{tabular}

the Aluminum stabilizer. It results in an increase of the normal zone propagation velocity around $17-18 \mathrm{kA}$. This is due to the shrinkage of the normal zone length $\left(L_{\mathrm{nzf}}\right)$ by increasing the magnet current. Therefore, it decreases the stability margins in this type of conductors considerably i.e., decreases the minimum quench energy.

\section{CONCLUSION}

The longitudinal normal zone propagation is measured inside the Atlas $B_{0}$ toroid model coil up to $0.25 I_{c}$. The typical values measured vary from 0.3 to $15 \mathrm{~m} / \mathrm{s}$ at 8 and $24 \mathrm{kA}$, respectively. A mathematical model, in order to describe the longitudinal normal zone propagation inside Aluminum stabilized superconductors has been developed. In the adiabatic case, the analytical solution can describe the normal zone propagation between 0 and the critical current $\left(I_{c}\right)$. The experimental results of the longitudinal propagation are compared to the mathematical results and a good correlation is found. In both cases, the propagation shows a change around a magnet current of 17-18 kA. By using the model, this mechanism of change can be understood because of the relatively long current diffusion compared to the longitudinal heat diffusion. It also predicts that the front of the normal zone does not occupy the complete stabilizing material, which reduces the stability margins in Aluminum stabilized superconductors considerably.

\section{REFERENCES}

[1] H. ten Kate, "Superconducting magnet system for the ATLAS detector at CERN," IEEE Trans. Appl. Superconduct., vol. 10, p. 347, Mar. 2000.

[2] A. Devred, "Investigation of current redistribution in superstabilized superconducting winding when switching to the normal state," J. Appl. Phys., vol. 65, no. 10, pp. 3963-3967, May 15, 1989.

[3] C. A. Luongo et al., "Current diffusion effects on the performance of large monolithic conductors," IEEE Trans. Magn., vol. 25, no. 2, Mar. 1989.

[4] A. Lee, R. H. Wands, and R. W. Fast, "Study of current redistribution in aluminum stabilized superconductor," Cryogenics, vol. 32, no. 10, pp. 863-866, 1992.

[5] M. N. Wilson, Superconducting Magnets. Oxford, U.K.: Clarendon, 1983.

[6] L. Rossi et al., IEEE Trans. Appl. Superconduct., vol. 10, pp. 369-372, Mar. 2000.

[7] K. Wada et al., "Development of high-strength and high-RRR aluminum-stabilized superconductor," IEEE Trans. on Appl. Supercond., vol. 10, no. 1, pp. 373-376, Mar. 2000.

[8] C. H. Edwards and D. E. Penny, Differential Equations and Boundary Value Problems, 2nd ed. Englewood Cliffs, NJ: Prentice Hall, 2000.

[9] M. Scherer and P. Turowski, "Investigation of the velocity of a normal-conducting zone in technical superconductors," Cryogenics, pp. 515-520, Sept. 1978.

[10] P. Miele et al., "ATLAS $B_{0}$ toroid model coil test at CERN," presented at the 17th Int. Conf. Magnet Technology. Geneva (CH) 24-28.09.2001. 